\title{
Phospholipid complex based nanoemulsion system for oral insulin delivery: preparation, in vitro, and in vivo evaluations
}

This article was published in the following Dove Press journal: International Journal of Nanomedicine

\author{
Xiong-Bin $\mathrm{Hu}^{\mathrm{l}-3}$ \\ Tian-Tian Tang ${ }^{1-3}$ \\ Yong-Jiang $\mathrm{Li}^{1-3}$ \\ Jun-Yong $\mathrm{Wu}^{\mathrm{l}-3}$ \\ Jie-Min Wang ${ }^{1-3}$ \\ Xin-Yi Liu ${ }^{1-3}$ \\ Da-Xiong Xiang ${ }^{1-3}$
}

'Department of Pharmacy, The Second Xiangya Hospital, Central South

University, Changsha, Hunan, 4100II,

People's Republic of China; ${ }^{2}$ Institute of

Clinical Pharmacy, Central South

University, Changsha 4I00II, Hunan,

People's Republic of China; ${ }^{3}$ Hunan

Provincial Engineering Research Center

of Translational Medicine and Innovative

Drug, Changsha, Hunan Province,

4100 II, People's Republic of China
Correspondence: Da-Xiong Xiang Department of Pharmacy, The Second Xiangya Hospital of Central South University, Changsha 4I00II, Hunan,

People's Republic of China

Tel +8673185292129

Email xiangdaxiong@csu.edu.cn
Purpose: The aim of this research was to develop a phospholipid complex based nanoemulsion system for oral insulin delivery.

Methods: Insulin-phospholipid complex (IPC) was firstly prepared by an anhydrous cosolvent lyophilization method, and then encapsulated into the oil phase of nanoemulsion to obtain the IPC-based nanoemulsion (IPC-NE). Both water-in-oil (W/O) IPC-NE and oil-inwater $(\mathrm{O} / \mathrm{W})$ IPC-NE were formulated and evaluated for comparison.

Results: The obtained W/O IPC-NE and O/W IPC-NE were both spherical in shape with a mean particle size of $18.6 \pm 0.79 \mathrm{~nm}$ and $27.3 \pm 1.25 \mathrm{~nm}$, respectively. While both IPC-NEs exhibited enhanced Caco-2 cell monolayers permeability than IPC and insulin solution, W/O IPC-NE showed relatively greater protective effects against enzymatic degradation than $\mathrm{O} / \mathrm{W}$ IPC-NE. Moreover, oral administration of W/O IPC-NE exhibited significant hypoglycemic effects, with 12.4-fold and 1.5-fold higher oral bioavailability compared with insulin solution and $\mathrm{O} / \mathrm{W}$ IPC-NE, respectively.

Conclusion: IPC-NEs, especially the W/O IPC-NE showed promising efficiency in vitro and in vivo, thus could be a potential strategy for oral insulin delivery.

Keywords: insulin, phospholipid complex, oral drug delivery, nanoemulsion, hypoglycemic effect

\section{Introduction}

Insulin is one of the most important therapeutic drugs for controlling blood glucose in patients with diabetes. Subcutaneous injection remains the main route for insulin delivery. However, discomfort and side effects such as pain, allergic reaction, lipohypertrophy, and hypoglycemia lead to low patient compliance. ${ }^{1}$ The oral route is considered as the natural and safest route for insulin administration because of convenience, non-invasion, and better glucose homeostasis. ${ }^{2}$ Nevertheless, the oral bioavailability and pharmacological action of insulin in the form of conventional solutions are far from desirable, mainly because of enzymatic degradation in the gastrointestinal tract (GT) and poor permeability across the intestinal membranes. ${ }^{3,4}$

Various delivery strategies have been developed to overcome these barriers, including liposomes, ${ }^{5}$ nanoemulsions, ${ }^{6}$ nanospheres, ${ }^{7}$ and nanoparticles. ${ }^{8}$ Therein, nanoemulsion is thought to be a promising approach with advantages of spontaneous formation, thermodynamic stability , and facilitated administration. ${ }^{9}$ Previous studies have shown that nanoemulsion can be utilized to improve permeation and oral bioavailability of many active ingredients, such as puerarin, ${ }^{10}$ dabigatran 
etexilate ${ }^{11}$, and paeonol. ${ }^{12}$ However, as a hydrophilic drug, insulin is always present in the aqueous phase. Therefore, insulin in oil-in-water $(\mathrm{O} / \mathrm{W})$ nanoemulsion would be destroyed by GT enzymes, and phase inversion may occur for water-in-oil (W/O) nanoemulsion as soon as the preparation diluted by a large amount of GT fluid, and lead to the destruction of insulin, with low oral bioavailability $(<0.2 \%){ }^{13}$

Previous studies have shown that oily vehicles could protect proteins from enzymatic degradation in the GT. ${ }^{14}$ Moreover, it would be helpful for proteins to absorb effectively when loaded inside nanosized oil droplets. ${ }^{15}$ However, as a highly hydrophilic protein, insulin cannot be loaded directly into the oil phase. Hence, the phospholipid complex method was utilized to improve the liposolubility of insulin to load into the oil phase of nanoemulsion in this study. As shown in previous studies, the formation of the drugphospholipid complex could improve the lipophilicity of the drugs. ${ }^{16,17}$ Moreover, Cui et al, demonstrated that insulin could be successfully complexed with phospholipid and solubilized within the hydrophobic phase. ${ }^{18}$ Therefore, we speculate that insulin-phospholipid complex (IPC) may facilitate the loading of insulin into the oil phase of the nanoemulsion, thus overcome the absorption barriers of oral insulin delivery and enhance its oral bioavailability.

In this study, O/W nanoemulsion and $\mathrm{W} / \mathrm{O}$ nanoemulsion were developed for oral insulin delivery, in which insulin was loaded in the oil phase by employed the phospholipid complex technique. The nanoemulsion systems were optimized with the aid of ternary phase diagrams, and characterized with respect to particle size, morphology, conductivity, and drug content. The selected IPC-based nanoemulsions (IPC-NEs) were evaluated in vitro with enzyme degradation studies and permeation experiments across Caco- 2 cell monolayers. Finally, the in vivo hypoglycemic effect and pharmacokinetics of IPCNEs via oral delivery were studied in streptozotocin (STZ)-induced diabetic rats.

\section{Materials and methods}

\section{Materials}

Porcine insulin (28.4 IU/mg) was purchased from Wanbang Biochemical (Jiangsu, China). Phospholipid was obtained from Taiwei Pharmaceutical Co., Ltd. (Shanghai, China). Oleic Acid (OA), Ethyl Oleate (EO), and Isopropyl Myristate (IPM) were purchased from Sinopharm Chemical Reagent Co., Ltd. (Shanghai, China). Cremophor EL35
(EL35), Transcutol HP and Cremophor RH40 (RH40) were purchased from BASF (Ludwigshafen, Germany). Pepsin, trypsin, 3-(4,5-dimethyl-2-thiazolyl)-2,5-diphenyl-2H-tetrazolium bromide (MTT), Hank's balanced salt solution (HBSS), and dimethyl sulfoxide (DMSO) were purchased from SigmaAldrich (Steinheim, Germany). Methanol and acetonitrile (HPLC grade) were provided by Tedia Company (USA). penicillin-streptomycin $(100 \mathrm{U} / \mathrm{mL})$ and ${ }_{\mathrm{L}}$-glutamine were purchased from Beyotime Biotechnology (Jiangsu, China). Fetal bovine serum (FBS), $0.25 \%$ trypsin-EDTA and Dulbelcco's Modified Eagle's Media (DMEM) were obtained from Fisher Scientific France (Illkirch, France). All other reagents employed in this study were of analytical grade.

\section{Preparation and characterization of IPC Preparation of IPC}

The IPC was prepared by an anhydrous co-solvent lyophilization method as previously described. ${ }^{18}$ Briefly, phospholipid and insulin powder (as a molar ratio of 1:1, 2:1, $4: 1,8: 1,16: 1,32: 1$, and $64: 1 \mathrm{w} / \mathrm{w}$, respectively) were dissolved in DMSO containing 5\% glacial acetic acid and then stirred to form a clear solution. The resultant solution was lyophilized overnight at $-40^{\circ} \mathrm{C}$ with a vacuum of $10 \mathrm{~Pa}$ using a condenser. The obtained IPC was firmly sealed for further experiments.

\section{Solubility study}

To verify whether the liposolubility of insulin was successfully improved in the form of IPC. Different oils (OA, EO, and IPM) were used to evaluate the altered solubility of insulin. Briefly, aliquots of oil $(1 \mathrm{~mL})$ were added into complexes or physical mixtures with different molar ratios of phospholipid vs insulin, followed by gentle vortex to reach equilibrium. The transmittance of each system was determined by an ultraviolet-visible spectrophotometer (UV-1780, SHIMADZU, Japan).

\section{Interaction between insulin and phospholipid}

X-ray diffractometry (D/max-r A; Rigaku Denki, Tokyo, Japan) and Fourier transform infrared spectrophotometry (FT-IR Spectrometer IFS-55, Switzerland) were utilized to study the interaction between insulin and phospholipid. The $\mathrm{X}$-ray diffractogram was scanned with the diffraction angle increasing from $5^{\circ}$ to $50^{\circ}, 2 \theta$ angle, with a stepwise angle of $0.04^{\circ}$ and a count time of $1 \mathrm{~s}$. The IR spectra of insulin, phospholipid, IPC and physical mixture of insulin and phospholipid were obtained by the $\mathrm{KBr}$ method. 


\section{Preparation of nanoemulsions}

Based on the results of the solubility study, EO was selected as the oil phase. The effects of surfactant (Lecithin, Labrasol for W/O nanoemulsion and EL35, RH40 for O/W nanoemulsion) and co-surfactant (Ethanol, propylene glycol for both $\mathrm{W} / \mathrm{O}$ and $\mathrm{O} / \mathrm{W}$ nanoemulsion) on the pseudo-ternary phase diagram were systematically observed at room temperature. The surfactant and co-surfactant were weighed at different ratios $(1: 2$, $1: 1,2: 1$, and $3: 1, w / w)$ and then vortexed vigorously for $30 \mathrm{~s}$ to obtain the surfactant mixture $(\mathrm{Sm})$. Afterwards, the oil and the Sm were mixed, where the ratios of oil to $\mathrm{Sm}$ in the mixtures were varied from 9:1 to $1: 9(\mathrm{w} / \mathrm{w})$ Distilled water was added dropwise to the mixture of oil and $\mathrm{Sm}$ at room temperature, with gentle stirring to allow equilibration. Following the addition of an aliquot of the water phase, the mixture was visually examined for phase transparency. The points from clear to turbid and turbid to clear were designated as emulsion and nanoemulsion, respectively.

\section{Preparation and characterization of IPC-NEs}

Based on the pseudo-ternary phase diagrams, the $\mathrm{W} / \mathrm{O}$ nanoemulsion formulation (Lecithin/Ethanol/EO/water= 26.67:26.67:35.55:11.11, w/w) and the $\mathrm{O} / \mathrm{W}$ nanoemulsion formulation (EL35/propylene glycol/EO/water $=18$. 18:6.06:6.06:69.70, w/w) were selected for further experiments. To formulate insulin-loaded nanoemulsion, IPC (insulin:phospholipid $=1: 32$ ) was added to EO, followed by gentle agitation to obtain the "drug loaded oil", then W/O IPC-NE and O/W IPC-NE were prepared to follow the same procedures of nanoemulsions preparation, with the exception of oil being replaced by the formulation amount of the "drug loaded oil", then the obtained IPC-NEs were purified by ultrafiltration (Amicon Millipore; $100 \mathrm{KDa}$ cut-off).

The morphology of the IPC-NEs was observed by transmission electron microscopy (TEM, JEOL JEM2100, Japan). Prior to the observation, samples were dropped on copper grids followed by drying at ambient temperature, then stained with $1 \%$ phosphotungstic acid solution. The electrical conductivity was determined at ambient temperature with a DDS-11A digital conductivity meter coupled to a platinum electrode (Shanghai Leida Instrument Co, Shanghai, China). The droplet size and polydispersity index (PDI) of IPC-NEs were determined by dynamic light scattering using a NANO-ZS90 particle size analyzer (Malvern Instruments, UK).

Insulin content in IPC-NEs was measured by the HPLC method. Briefly, $0.5 \mathrm{~mL}$ of IPC-NEs was diluted to $5 \mathrm{~mL}$ with $1 \%$ acetic acid-methanol, then the solution samples $(20 \mu \mathrm{L})$ were analyzed by HPLC system (Agilent 1200) with the following conditions: Hypersil C18 column $(4.6 \times 200 \mathrm{~mm}, 5$ $\mu \mathrm{m})$, the mobile phase consisted of acetonitrile and $0.2 \mathrm{M} \mathrm{Na}_{2}$ $\mathrm{SO}_{4}$ solution adjusted to $\mathrm{pH} 2.3$ with phosphoric acid (30:70, $\mathrm{v} / \mathrm{v}$ ) at a flow rate of $1.0 \mathrm{~mL} / \mathrm{min}$. The detector wavelength was set at $214 \mathrm{~nm}$ and the column temperature was maintained at $35^{\circ} \mathrm{C}$. The entrapment efficiency (EE) was calculated according to the following equation: $\mathrm{EE} \%=M_{\mathrm{in}} / M_{\text {total }} \times 100 \%$ (where $M_{\mathrm{in}}=$ the entrapped insulin in IPC-NEs, $M_{\mathrm{total}}=$ the total amount of drug). The stability of W/O IPC-NE and O/ W IPC-NE was investigated via appearance, droplet size, and insulin content at ambient temperature and $4{ }^{\circ} \mathrm{C}$ up to 3 months.

\section{Drug release and enzyme degradation study}

The release profiles of insulin from IPC-NEs were investigated via dialysis method. Briefly, $2 \mathrm{~mL}$ of IPC-NEs was added into the dialysis tubing (MWCD: $100 \mathrm{kDa}$ ), and submerged in $1,000 \mathrm{~mL}$ of simulated gastric fluid (SGF, $\mathrm{pH} 2.0$ ) for $2 \mathrm{hrs,}$ then the medium was replaced by equal volume of simulated intestinal fluid (SIF, pH 6.8) for incubation of $6 \mathrm{hrs}$. The dialysis devices were under magnetic stirring at $37^{\circ} \mathrm{C}$ during the release study. At predetermined time points, samples (200 $\mu \mathrm{L}$ ) were withdrawn and the remaining insulin was analyzed by HPLC method as mentioned above.

To evaluate the protective effect of IPC-NEs against enzymatic degradation, W/O IPC-NE and O/W IPC-NE were dispersed in SGF containing pepsin $(0.05 \mathrm{mg} / \mathrm{mL})$ or SIF containing trypsin $(3 \mathrm{mg} / \mathrm{mL})$ respectively. The mixture was immediately incubated at $37^{\circ} \mathrm{C}$. Aliquots of the mixture $(200 \mu \mathrm{L})$ were withdrawn at regular intervals and added to an equal volume of $0.1 \mathrm{M} \mathrm{NaOH}$ for SGF, $0.1 \mathrm{M}$ HCL for SIF to stop enzymatic activity. ${ }^{19}$ The amount of remaining insulin was analyzed using HPLC as described above. IPC and free insulin were also tested as a control.

\section{Transport experiment with Caco-2 cell monolayers \\ Cell culture}

Caco-2 cells were obtained from Shanghai Institutes for Biological Sciences, Chinese Academy of Sciences 
(Shanghai, China). The cells were cultured in DMEM supplemented with $15 \% \mathrm{FBS}, 1 \%$ penicillin-streptomycin $(100 \mathrm{U} / \mathrm{mL})$ and $1 \% \mathrm{~L}$-glutamine under $5 \% \mathrm{CO}_{2}$ atmosphere condition at $37^{\circ} \mathrm{C}$.

\section{MTT cytotoxicity assay}

Caco-2 cells were seeded on 96-well plates at a density of $6.5 \times 10^{4}$ cells/well and cultured at $37^{\circ} \mathrm{C}$ for $24 \mathrm{hrs}$. The cells were then incubated with different concentrations $(10,25,50,100 \mu \mathrm{g} / \mathrm{mL})$ of test samples (insulin solution or insulin formulations) for $4 \mathrm{hrs}$. After that, $20 \mu \mathrm{L}$ of MTT solution $(5 \mathrm{mg} / \mathrm{mL})$ was added to each well $\left(37^{\circ} \mathrm{C}, 5 \% \mathrm{CO}_{2}\right)$. After $4 \mathrm{hrs}$ of incubation, the media was removed and DMSO $(150 \mu \mathrm{L})$ was added to dissolve the formazan precipitate. The formazan was quantified by measuring the optical density to calculate cell viability according to a previous report. ${ }^{20}$

\section{Transport study}

To evaluate the permeability of W/O IPC-NE, O/W IPC-NE, IPC or free insulin across Caco-2 cell monolayers, Caco- 2 cells were seeded at a density of $1 \times 10^{5}$ cells/well on 12-well Transwell ${ }^{\circledR}$ culture plates (Corning Costar, USA) and grown for 21 days. Transepithelial electrical resistance (TEER) of the monolayer was measured before and after the experiment using a Millicell-Electrical Resistance System (Endohm-12, Millipore Corp). The monolayers with the initial TEER higher than $>450 \Omega \mathrm{cm}^{2}$ were used for further transport experiments. One hour prior to the transport experiment, the culture medium was replaced with an equal volume of HBSS. After equilibration, the donor chamber medium was replaced by fresh medium containing W/O IPC-NE, O/W IPC-NE, IPC, or free insulin $(100 \mu \mathrm{g} / \mathrm{mL})$. Aliquots were withdrawn from the receiver chamber at different time intervals for 3 hrs, and the samples were analyzed by HPLC. The apparent permeability coefficient $\left(P_{a p p}\right)$ of insulin was calculated using the following equation:

$$
P_{a p p}=\frac{d Q}{d t} \times \frac{1}{A \cdot C_{0}}
$$

where $d Q / d t$ is the permeability rate, $A$ is the surface area of the membrane $\left(\mathrm{cm}^{2}\right), C_{0}$ is the initial concentration in the apical chamber. Absorption enhancement ratio $(R)$ was calculated as follows:

$$
R=\frac{P_{a p p}(\text { sample })}{P_{\text {app }}(\text { control })}
$$

\section{In vivo evaluation of IPC-NEs}

Bioactivity of encapsulated insulin

To evaluate the bioactivity of insulin after the production process, the hypoglycemic effect of the released insulin from IPC-NEs or IPC was evaluated in normal rats. Briefly, insulin released from IPC-NEs or IPC at $37^{\circ} \mathrm{C}$ was collected and the concentration was determined by HPLC. Male Sprague-Dawley (SD) rats (200-250 g) (fasted for $16 \mathrm{hrs}$ with free access to water) were injected subcutaneously with the released insulin or free insulin at a dose of $1 \mathrm{IU} / \mathrm{kg}(\mathrm{n}=6)$. The blood glucose level was determined using the Roche ACCU-CHEK Go (Basel, Switzerland) glucose-meter at the predesigned time point.

\section{Pharmacological and pharmacokinetics study}

Diabetic animal models were developed in adult male SD rats induced by single intraperitoneal injection of STZ $(80 \mathrm{mg} / \mathrm{kg})$ as previously described. ${ }^{21}$ Only the rats with the baseline glucose levels over $250 \mathrm{mg} / 100 \mathrm{~mL}$ were considered as diabetic. The diabetic rats were fasted for $16 \mathrm{hrs}$ with free access to water prior to the experiments. Rats were divided into five groups ( $\mathrm{n}=6$ per group). (i) $\mathrm{W} /$ O IPC-NE, (ii) O/W IPC-NE, (iii) IPC, and (iv) free insulin were administered orally at an insulin dose of 50 $\mathrm{IU} / \mathrm{kg}$. (v) Insulin solution was also subcutaneously injected (s.c.) at a dose of $1 \mathrm{IU} / \mathrm{kg}$. Blood samples were collected from the tail vein prior to administration and at different time points post administration. Blood glucose levels were determined by the Roche ACCU-CHEK Go (Basel, Switzerland) glucose meter. Plasma insulin levels were measured by a porcine insulin ELLSA kit (R\&D System, USA). Area under the curve (AUC) of plasma insulin concentration was computed, and the relative bioavailability $(F \%)$ was calculated as follows:

$$
\mathrm{F}(\%)=\frac{\mathrm{AUC}_{\text {oral }} \times \text { Dose }_{\text {s.c. }}}{\mathrm{AUC}_{\text {s.c. }} \times \text { Dose }_{\text {oral }}} \times 100 \%
$$

Male SD rats (200-250 g) were obtained from Hunan Slack Scene of Laboratory Animal Co., Ltd. All animal studies were performed in accordance with the Guide for Care and Use of Laboratory Animal, and all procedures were approved by Central South University Ethics Committee (Protocol number SYXK-2012-003).

\section{Statistics}

All data were presented as mean \pm standard deviation (SD) and analyzed using SPSS 19.0 (SPSS, Chicago, IL). Difference between groups was evaluated using 
the two-tailed Student's $t$-test. AP -value of less than 0.05 was considered statistically significant.

\section{Results and discussion Characterization of IPC}

Figure 1 shows the effects of phospholipid/insulin molar ratio on the solubilization of IPC in different oil media. With the increase of the molar ratio of phospholipid vs insulin in complex, the transmittance of the oil media increased gradually. At the ratio of 32:1, the transmittance reached its maximum, and the transmittance in EO reached about $100 \%$, indicating that insulin was successfully dissolved in EO. However, the transmittance of the corresponding physical mixture was less than $20 \%$, suggesting that insulin could not be solubilized effectively in oils by simply mixing with phospholipid.

Figure 2A shows the X-ray diffraction patterns of insulin, phospholipid, their physical mixture, and IPC. There were partial sharp crystalline peaks in the diffraction pattern of insulin powder, which is the characteristic of a macromolecule with some crystalline structure. ${ }^{22}$ In contrast, phospholipid appeared to be an amorphous structure. In the profile of physical mixture, the crystalline signal of insulin remained unchanged. On the contrary, the crystalline peaks were disappeared in IPC, showing a structural characteristic similar to that of phospholipid. This might be because of noncovalent interaction between insulin and

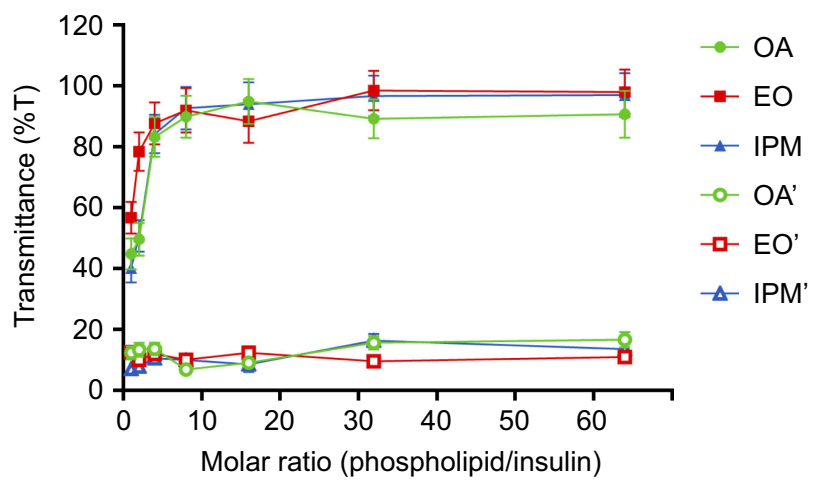

Figure I The effect of molar ratio (phospholipids/insulin) on the solubilization of IPC. Complex in oils (OA, EO, and IPM); physical mixture in oils (OA', EO', and IPM'). Data are shown as mean $\pm S D, n=3$.

Abbreviations: EO, ethyl oleate; IPM, isopropyl myristate; IPC, insulinphospholipid complex; OA, oleic acid.

phospholipid, and insulin was likely to be molecularly dispersed in IPC.

The IR spectra of insulin, phospholipid, the physical mixture, and IPC were shown in Figure 2B. The physical mixture and the complex showed distinct IR spectra. The characteristic absorption peaks of insulin were still present at 1,728 and $1,653 \mathrm{~cm}^{-1}$ in the spectrum of physical mixture. However, in the spectrum of IPC, the two characteristic absorption peaks of insulin were almost masked by that of phospholipid. Moreover, no new peaks were observed in the spectrum of physical mixture and IPC.
A

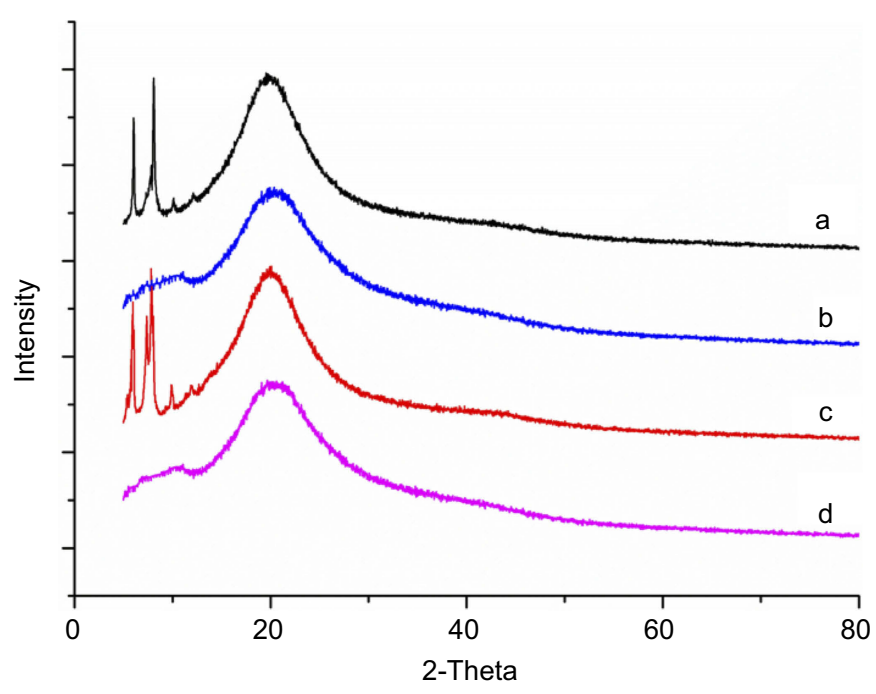

B

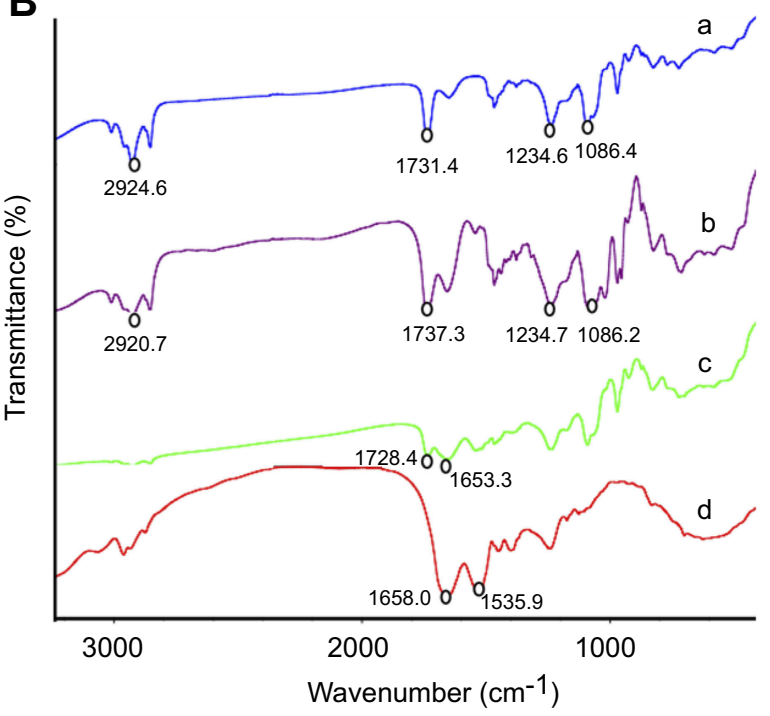

Figure 2 (A) X-ray diffraction patterns of (a) insulin, (b) phospholipid, (c) physical mixture of insulin and phospholipid, (d) IPC; (B) infrared spectra of (a) phospholipid, (b) IPC, (c) physical mixture of insulin and phospholipid, (d) insulin.

Abbreviation: IPC, insulin-phospholipid complex. 


\section{Preparation and characterization of IPC-NEs}

Based on the results of the solubility study of IPC, EO was selected as the oil phase of nanoemulsion. Pseudo-ternary phase diagrams were constructed to obtain the appropriate concentration ranges of other components of nanoemulsion. The constructed phase diagrams were described in Figure S1. The nanoemulsion region was present as a gray zone in the phase diagrams. From the results obtained here together with the appearance and stability, W/O nanoemulsion $($ Lecithin/Ethanol/EO/water=26.67:26.67:35.55:11.11, $\mathrm{w} / \mathrm{w}$ ) and $\mathrm{O} / \mathrm{W}$ nanoemulsion (EL35/propylene glycol/EO/ water $=18.18: 6.06: 6.06: 69.70, \mathrm{w} / \mathrm{w})$ were selected as the optimized preparations. Insulin was then incorporated to the selected formulations according to the method described in this paper to obtain W/O IPC-NE and O/W IPC-NE for further investigation.

The particle size of O/W IPC-NE and W/O IPC-NE was $18.6 \pm 0.79$ and $27.3 \pm 1.25 \mathrm{~nm}$, respectively. The PDI value were both less than 0.5 , which indicates the homogeneity of the nanoemulsion formulations. Insulin content of O/W IPC$\mathrm{NE}$ was $216.5 \pm 0.57 \mathrm{mg} / \mathrm{mL}$ with an $\mathrm{EE} \%$ of $89.4 \pm 1.5 \%$, and the insulin concentration in W/O IPC-NE was 769.78 $\pm 0.86 \mathrm{mg} / \mathrm{mL}$ with an $\mathrm{EE} \%$ of $91 \pm 2.2 \%$. Their respective conductivities were $164.9 \pm 3.92$ and $24.7 \pm 1.03 \mu \mathrm{s} / \mathrm{cm}$. The TEM images of the prepared IPC-NEs are shown in Figure 3. Obviously, O/W IPC-NE and W/O IPC-NE both appeared spherical morphology with a particle size smaller than 50 $\mathrm{nm}$, which was in accordance with the results obtained from particle size analyzer. O/W IPC-NE and W/O IPC-NE were stable at ambient temperature and $4^{\circ} \mathrm{C}$ up to 3 months, indicated that these formulations were suitable for further experiments in our study (Table S1).

\section{Drug release and enzyme degradation study}

To imitate the GT environment, we conducted in vitro release study in SGF for the first $2 \mathrm{hrs}$ and in SIF for another $6 \mathrm{hrs}$, without enzymes. ${ }^{23}$ As illustrated in Figure 4A, both of free insulin and IPC had a fast release profile, with more than $80 \%$ of the insulin were released within the first $2 \mathrm{hrs}$. In comparison, IPC-NEs demonstrated sustained release profiles in both SGF and SIF media. The cumulative release of insulin within 2 hrs from O/W IPC-NE and W/O IPC-NE was $61 \%$ and 49\%, respectively. We found that the type of nanoemulsion plays an important role in controlling the drug release. After $8 \mathrm{hrs}$ of drug release, about $70 \%$ of the loaded insulin was released from W/O IPC-NE, which was much lower than that of O/W IPC-NE ( $\sim 90 \%)$.

The protection ability of the prepared IPC-NEs on the entrapped insulin against digestive enzymes was investigated in vitro under the presence of pepsin or trypsin. Figure 4B shows the remaining percentage of insulin in IPC-NEs or IPC after incubation with pepsin. As a result, the protective effect was as follows: W/O IPC-NE $>$ O/W IPC-NE $>$ IPC $>$ insulin. Insulin was rapidly and completely destroyed without the protection of nanocarriers by pepsin within $1 \mathrm{hr}$. In contrast, $18.6 \%$ and $4.8 \%$ of insulin were preserved in W/O IPC-NE
A

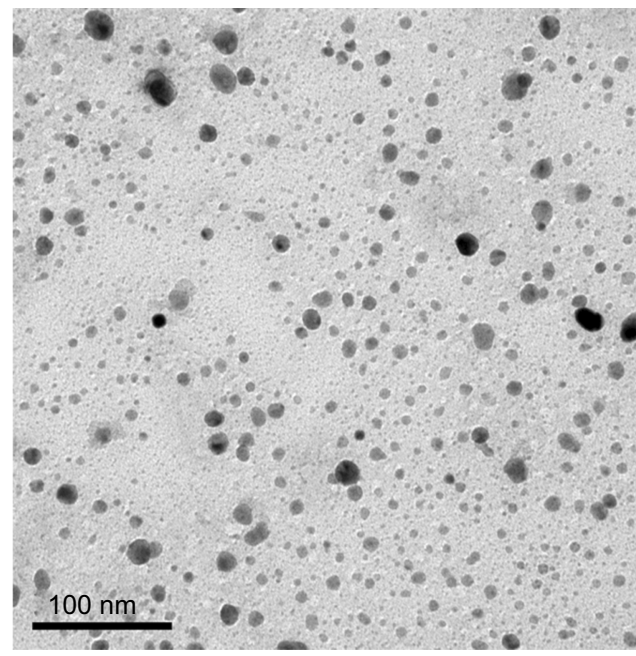

B

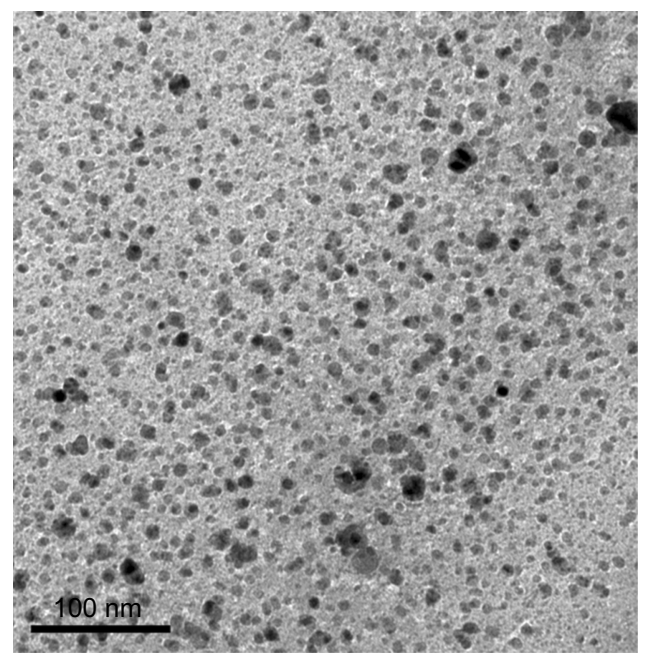

Figure 3 Transmission electron microphotography (TEM) of IPC-NEs: (A) O/W IPC-NE and (B) W/O IPC-NE.

Abbreviations: IPC, insulin-phospholipid complex; IPC-NE, IPC-based nanoemulsion; O/W, oil-in-water; W/O, water-in-oil. 
A

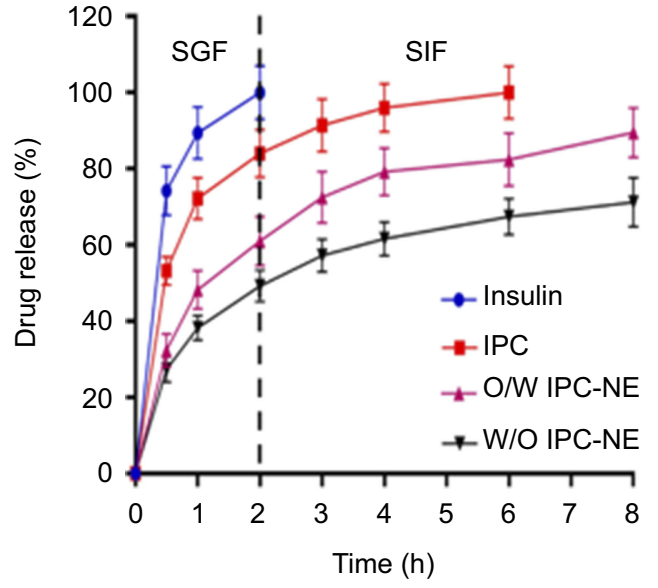

B

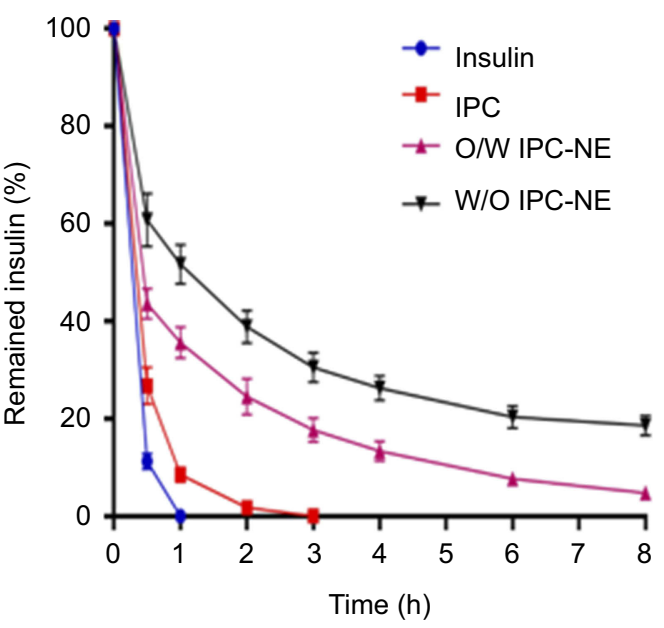

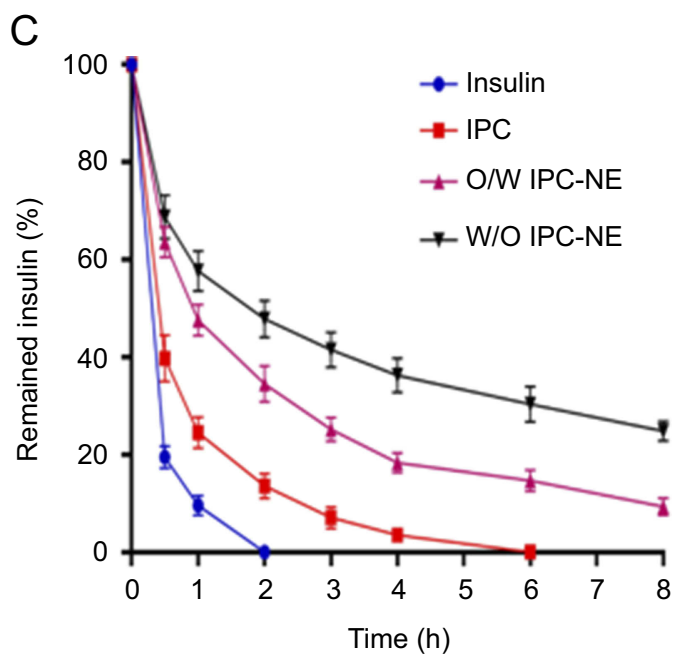

Figure 4 (A) In vitro release profiles of insulin from IPC-NEs or IPC in SGF for 2 hrs and SIF for 6 hrs; (B) percentage of insulin remaining after incubation of different samples with pepsin in SGF; (C) percentage of insulin remaining after incubation of different samples with trypsin in SIF. Data are shown as mean \pm SD, $n=3$.

Abbreviations: IPC, insulin-phospholipid complex; IPC-NE, IPC-based nanoemulsion; O/W, oil-in-water; SGF, simulated gastric fluid; SIF, simulated intestinal fluid; W/O, water-in-oil.

and O/W IPC-NE after 8 hrs. The prepared formulations showed a similar protective effect of insulin against trypsin and pepsin as shown in Figure 4C. The amount of intact insulin in W/O IPC-NE and O/W IPC-NE were $24.9 \%$ and 9.4\% after $8 \mathrm{hrs}$ incubation with trypsin, respectively. W/O IPC-NE showed a stronger protective effect against enzyme degradation as compared to O/W IPC-NE and IPC. These protective patterns may be attributed to the different release profiles of each system. These results were also consistent with a previous report that the external oily phase was important in the protection of insulin against enzymatic degradation. ${ }^{14}$

\section{Transport through Caco-2 cell monolayers}

MTT cytotoxicity assay was carried out to evaluate the toxicity of insulin formulations on Caco-2 cells. As shown in Figure S2, no significant cytotoxicity was observed at the concentration from 10 to $100 \mu \mathrm{g} / \mathrm{mL}$ in all of the treated groups (cell viability $>90 \%$ ). Thus, $100 \mu \mathrm{g} / \mathrm{mL}$ was selected as the working concentration in the transport experiments.

Caco-2 cell monolayers were utilized to study the permeation effect of IPC-NEs. Both the TEER values before and after the transport experiments were higher than $>450$ $\Omega \mathrm{cm}^{2}$, confirmed the integrity of the cell monolayers. ${ }^{20}$ The amount of transported insulin from each formulation was as follows: IPC-NEs $>$ IPC $>$ insulin (Figure 5). The $P_{a p p^{-}}$ values of insulin from each formulation were compared, and results are summarized in Table 1. The permeation of insulin across Caco-2 cell monolayers was improved by IPC, representing a 1.26-fold enhancement of the insulin permeation coefficient. This may be the result of increased 


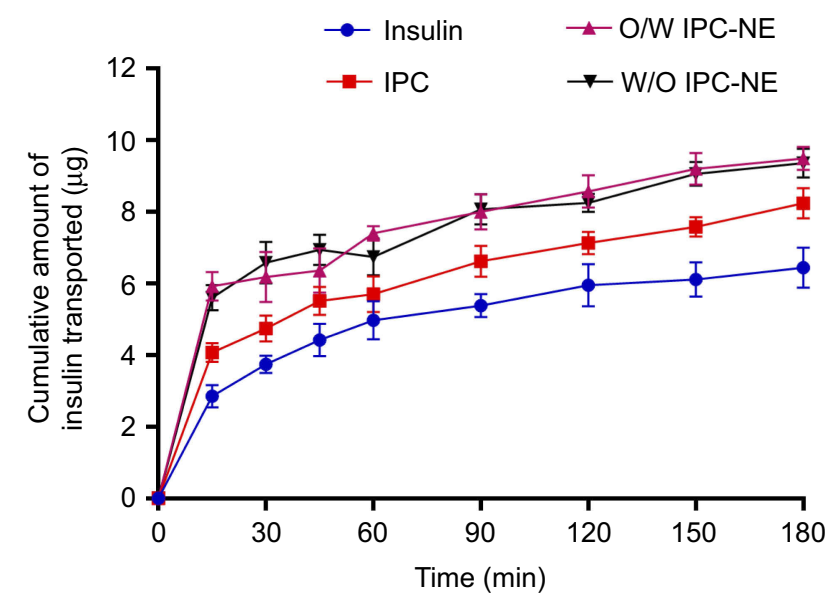

Figure 5 Cumulative amount of insulin transported across Caco-2 cell monolayers at $37^{\circ} \mathrm{C}$. Data are shown as mean $\pm S D, n=3$.

Abbreviations: IPC, insulin-phospholipid complex; IPC-NE, IPC-based nanoemulsion; O/W, oil-in-water; W/O, water-in-oil.

Table I Apparent permeability coefficient and absorption enhancement ratio for insulin across Caco-2 cell monolayers (mean $\pm \mathrm{SD}, \mathrm{n}=3$ )

\begin{tabular}{|l|l|l|}
\hline Formulation & $\boldsymbol{P}_{\boldsymbol{a p p}} \times 1 \mathbf{0}^{-6} \mathbf{~ c m} / \mathbf{s}$ & $\boldsymbol{R}$ \\
\hline Insulin (control) & $12.1 \pm 0.3$ & - \\
IPC & $15.3 \pm 0.5^{*}$ & 1.26 \\
W/O IPC-NE & $17.9 \pm 0.2^{*, \#}$ & 1.48 \\
O/W IPC-NE & $18.1 \pm 0.3^{*, \#}$ & 1.50 \\
\hline
\end{tabular}

Notes: ${ }^{*} \mathrm{P}<0.05$ compared with insulin (control), ${ }^{\#} \mathrm{P}<0.05$ compared with IPC. Abbreviations: $P_{a p p}$, the apparent permeability coefficient; $R$, absorption enhancement ratio; IPC, insulin-phospholipid complex; IPC-NE, IPC-based nanoemulsion; O/W, oil-in-water; W/O, water-in-oil.

lipophilicity in IPC. Moreover, as an important component of cell membrane, phospholipid can improve drug transportation across the Caco- 2 cells. ${ }^{17}$ It was shown that the largest amount of insulin transport was facilitated by IPC-NEs. The permeability coefficients of IPC-NEs were significantly higher than that of insulin $(P<0.05)$, representing 1.48 -fold and 1.5-fold enhancement for W/O IPC-NE and O/W IPC$\mathrm{NE}$, respectively. While there was no significant difference between W/O IPC-NE and O/W IPC-NE $(P>0.05)$.

Nanoemulsion carriers played an important role in the enhancement of insulin permeation across Caco- 2 cell monolayers. A higher $P_{a p p}$-value was obtained with IPC-NEs in comparison with IPC in this study $(P<0.05)$. Numerous studies indicate that nanoemulsion has a significant potential to increase the permeability of drugs. The proposed mechanisms were based on surfactant induced structural and fluidity changes in the membranes. ${ }^{24,25}$ IPC-NEs combined phospholipid complex technique with nanoemulsion system, exhibited synergic effect of nanoemulsion and IPC on the transport properties of insulin across the Caco-2 cell monolayers.

\section{In vivo evaluation of IPC-NEs}

To confirm the maintained biological activity of insulin after encapsulated into IPC-NEs, the hypoglycemic effects of the released insulin were evaluated in normal rats according to a previous report. ${ }^{23}$ After subcutaneous injection, the released insulin from IPC-NEs and IPC showed similar hypoglycemic profiles as compared to the equivalent dose of free insulin (1 IU/kg; Figure 6A). There was no significant difference between the hypoglycemic effects of these groups $(P>0.05)$, suggesting that the bioactivity of insulin was maintained after encapsulation and release.

The hypoglycemic effect and pharmacokinetics of different insulin formulations were assessed in STZ-induced diabetic rats in vivo. As illustrated in Figure 6B, the blood glucose was reduced sharply to the minimum concentration (about $40 \%$ of the initial level) $2 \mathrm{hrs}$ after injection (insulin solution s.c., $1 \mathrm{IU} / \mathrm{kg}$ ), then gradually returned to the basal level within $8 \mathrm{hrs}$. Oral delivery of insulin solution failed to produce hypoglycemic effects. In contrast, oral administration of W/O IPC-NE, O/W IPC-NE, and IPC at an equivalent insulin dose $(50 \mathrm{IU} / \mathrm{kg})$ all resulted in reduction of the blood glucose level, the hypoglycemic effects of the preparations were as follows: W/O IPC-NE $>\mathrm{O} / \mathrm{W}$ IPC-NE $>$ IPC, the lowest percent of glucose level were $65.7 \%, 70.8 \%$, and $82.0 \%$, respectively.

The pharmacokinetic profiles of insulin and the related parameters were shown in Figure 6C and Table 2, respectively. Consistent with the hypoglycemic effect, there was only a slight serum insulin concentration increase in the IPC group as compared to oral insulin. In comparison, the maximal serum insulin concentration was significantly improved in W/O IPC$\mathrm{NE}$ and $\mathrm{O} / \mathrm{W}$ IPC-NE groups, which were 2.7 and 1.9-fold to that of IPC group $(P<0.05)$. W/O IPC-NE exhibited the highest oral bioavailability $(F \%)$ of $4.7 \%$, which was 12.4 -fold higher than that of oral insulin solution $(0.38 \%), \sim 1.7$-fold higher than that of IPC (2.8\%), and $\sim 1.5$-fold higher than that of O/W IPCNE (3.2\%), respectively.

The observed better in vivo hypoglycemic effects of IPCNEs than IPC may be attributed to its better protection and permeation achieved by IPC-NE systems as shown in enzyme degradation and Caco-2 cell monolayers permeation experiments. Furthermore, W/O IPC-NE showed stronger hypoglycemic effects compared to O/W IPC-NE, suggesting that type of nanoemulsion have a significant influence on oral absorption of insulin. This result was in accordance with the enzyme 
A

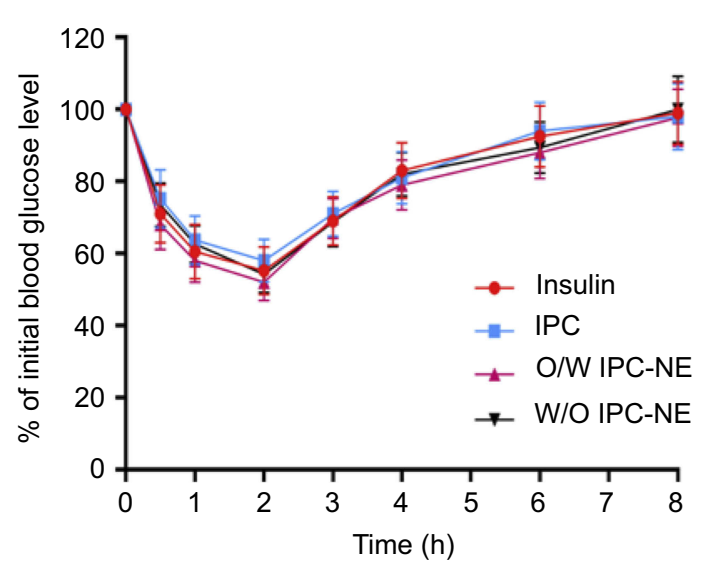

C
B

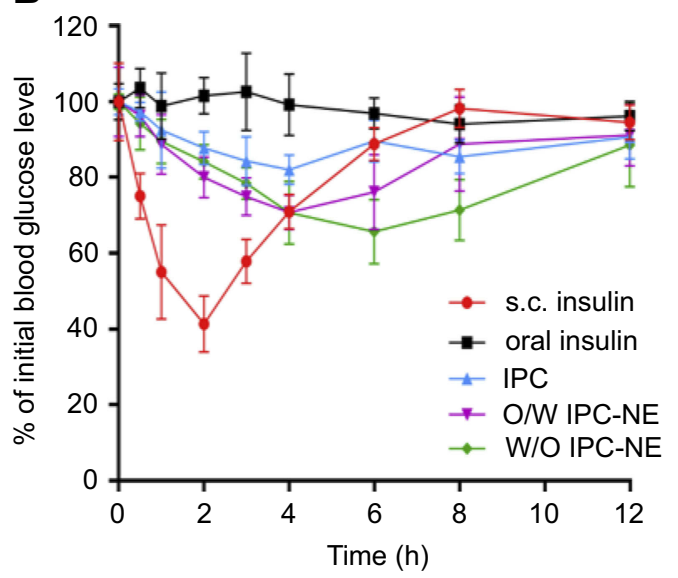

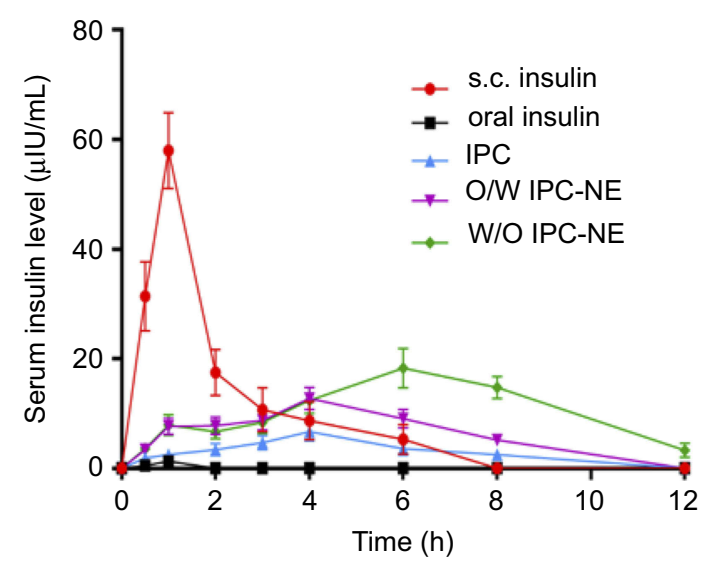

Figure 6 (A) Blood glucose level of normal rats after subcutaneous injection of equivalent dose (I IU/kg) of insulin solution or insulin released from IPC-NEs or IPC; (B) blood glucose levels in diabetic rats after oral administration of W/O IPC-NE, O/W IPC-NE, IPC and insulin solution (oral insulin, 50 IU/kg) and subcutaneous injection of insulin solution (s.c. insulin, I IU/kg); (C) serum insulin concentration in diabetic rats following administration of different formulations. Data are shown as mean \pm SD, $\mathrm{n}=6$. Abbreviations: IPC, insulin-phospholipid complex; IPC-NE, IPC-based nanoemulsion; O/W, oil-in-water; s.c., subcutaneous injection; W/O, water-in-oil.

Table 2 Pharmacokinetic parameters of insulin in diabetic rats $($ mean $\pm S D, n=6)$

\begin{tabular}{|l|l|l|l|}
\hline Sample & $\begin{array}{l}\text { Dose } \\
\text { (IU/kg) }\end{array}$ & $\begin{array}{l}\text { AUC } \\
\text { (mIU.h/L) }\end{array}$ & $\boldsymbol{F ~ ( \% ) ~}$ \\
\hline Free insulin (s.c.) & 1 & $58.19 \pm 6.37$ & 100 \\
Free insulin (oral) & 50 & $9.12 \pm 3.52$ & $0.38 \pm 0.12$ \\
IPC (oral) & 50 & $81.47 \pm 8.16$ & $2.8 \pm 0.28$ \\
O/W IPC-NE (oral) & 50 & $93.10 \pm 8.95$ & $3.2 \pm 0.31$ \\
W/O IPC-NE (oral) & 50 & $136.75 \pm 10.82$ & $4.7 \pm 0.37$ \\
\hline
\end{tabular}

Abbreviations: AUC, area under the curve of plasma insulin concentration; $F(\%)$, relative bioavailability $(n=6)$; IPC, insulin-phospholipid complex; IPC-NE, IPC-based nanoemulsion; O/W, oil-in-water; s.c., subcutaneous injection; W/O, water-in-oil.

degradation data that W/O IPC-NE had a better protective effect on insulin than O/W IPC-NE. In addition, sustained hypoglycemic effects were observed in the diabetic rats treated with W/O IPC-NE, suggesting that this delivery strategy may have promising potential as an oral basal insulin. ${ }^{26}$ This study demonstrated that IPC-NEs, especially the W/O IPC-NE, are promising vehicles for oral delivery of insulin, with excellent hypoglycemic effects and improved pharmacokinetic profiles.

\section{Conclusions}

In conclusion, the IPC-NEs, where insulin was loaded in the oil phase by employed a phospholipid complex technique, were successfully prepared. Both W/O IPC-NE and O/W IPC-NE significantly enhanced the protective effects of insulin against enzymes degradation and improved insulin transport across Caco-2 cell monolayers in vitro. Oral administration of IPC-NEs, especially the W/O IPC-NE showed significant and sustained hypoglycemic effect and higher oral bioavailability than that of oral insulin solution in diabetic rats. Our study demonstrated that the combined use of nanoemulsion and phospholipid complex technique 
might be a promising strategy for the development of oral formulations of peptide and protein drugs such as insulin.

\section{Acknowledgments}

This study was supported by the Nature Science Foundation of Hunan Province of China [2019JJ50882] and the Hunan Provincial Science and Technology Plan [2016TP2002].

\section{Disclosure}

The authors report no conflicts of interest in this work.

\section{References}

1. Saaddine JB, Cadwell B, Gregg EW, et al. Improvements in diabetes processes of care and intermediate outcomes: United States, 1988-2002. Ann Intern Med. 2006;144(7):465-474.

2. Owens DR, Zinman B, Bolli G. Alternative routes of insulin delivery. Diabetic Medicine. 2003;20(11):886-898.

3. Li P, Nielsen HM, Mullertz A. Oral delivery of peptides and proteins using lipid-based drug delivery systems. Expert Opin Drug Deliv. 2012;9(10):1289-1304. doi:10.1517/17425247.2012.717068

4. Rai VK, Mishra N, Agrawal AK, Jain S, Yadav NP. Novel drug delivery system: an immense hope for diabetics. Drug Deliv. 2016;23(7):2371-2390. doi:10.3109/10717544.2014.991001

5. Cui M, Wu W, Hovgaard L, Lu Y, Chen D, Qi J. Liposomes containing cholesterol analogues of botanical origin as drug delivery systems to enhance the oral absorption of insulin. Int J Pharm. 2015;489 (1-2):277-284. doi:10.1016/j.ijpharm.2015.05.006

6. Sharma G, Wilson K, van der Walle CF, Sattar N, Petrie JR, Ravi Kumar MN. Microemulsions for oral delivery of insulin: design, development and evaluation in streptozotocin induced diabetic rats. Eur JPharm Biopharm. 2010;76(2):159-169. doi:10.1016/j.ejpb.2010.07.002

7. Shi K, Fang Y, Kan Q, Zhao J, Gan Y, Liu Z. Surface functional modification of self-assembled insulin nanospheres for improving intestinal absorption. Int $J$ Biol Macromol. 2015;74:49-60. doi:10.1016/j.ijbiomac.2014.11.013

8. Woitiski CB, Neufeld RJ, Veiga F, Carvalho RA, Figueiredo IV. Pharmacological effect of orally delivered insulin facilitated by multilayered stable nanoparticles. Eur J Pharm Sci. 2010;41(3-4):556-563. doi:10.1016/j.ejps.2010.08.009

9. Li Y-J, Hu X-B, Lu X-L, et al. Nanoemulsion-based delivery system for enhanced oral bioavailability and Caco-2 cell monolayers permeability of berberine hydrochloride. Drug Deliv. 2017;24:1.

10. Wu J-Y, Li Y-J, Han M, et al. A microemulsion of puerarin-phospholipid complex for improving bioavailability: preparation, in vitro and in vivo evaluations. Drug Dev Ind Pharm. 2018;44(8):1336-1341.

11. Ge L, He X, Zhang Y, et al. A dabigatran etexilate phospholipid complex nanoemulsion system for further oral bioavailability by reducing drug-leakage in the gastrointestinal tract. Nanomedicine. 2018;14(4):1455-1464. doi:10.1016/j.nano.2017.08.009

12. Chen S, Zhang J, Wu L, Wu H, Dai M. Paeonol nanoemulsion for enhanced oral bioavailability: optimization and mechanism. Nanomedicine. 2018;13(3):269-282. doi:10.2217/nnm-2017-0277
13. Cilek A, Celebi N, Tirnaksiz F, Tay A. A lecithin-based microemulsion of rh-insulin with aprotinin for oral administration: investigation of hypoglycemic effects in non-diabetic and STZ-induced diabetic rats. Int J Pharm. 2005;298(1):176-185. doi:10.1016/j.ijpharm.2005.04.016

14. Elsayed A, Al Remawi M, Qinna N, Farouk A, Badwan A. Formulation and characterization of an oily-based system for oral delivery of insulin. Eur J Pharm Biopharm. 2009;73(2):269-279. doi:10.1016/j.ejpb.2009.06.004

15. Rao SVR, Yajurvedi K, Shao J. Self-nanoemulsifying drug delivery system (SNEDDS) for oral delivery of protein drugs III. In vivo oral absorption study. Int $J$ Pharm. 2008;362(1-2):16-19. doi:10.1016/j. ijpharm.2008.05.015

16. Qin L, Niu Y, Wang Y, Chen X. Combination of phospholipid complex and submicron emulsion techniques for improving oral bioavailability and therapeutic efficacy of water-insoluble drug. Mol Pharmaceut. 2018;15(3):1238-1247. doi:10.1021/acs.molpharmaceut.7b01061

17. Zhao YQ, Wang LP, Ma C, Zhao K, Liu Y, Feng NP. Preparation and characterization of tetrandrine-phospholipid complex loaded lipid nanocapsules as potential oral carriers. Int $J$ Nanomedicine. 2013;8:4169-4181. doi:10.2147/IJN.S50557

18. Cui F, Shi K, Zhang LQ, Tao AJ, Kawashima Y. Biodegradable nanoparticles loaded with insulin-phospholipid complex for oral delivery: preparation, in vitro characterization and in vivo evaluation. J Control Release. 2006;114(2):242-250. doi:10.1016/j. jconrel.2006.05.013

19. Niu Z, Samaridou E, Jaumain E, et al. PEG-PGA enveloped octaarginine-peptide nanocomplexes: an oral peptide delivery strategy. J Control Release. 2018;276:125-139. doi:10.1016/j. jconrel.2018.03.004

20. Metre S, Mukesh S, Samal SK, Chand M, Sangamwar AT. Enhanced biopharmaceutical performance of rivaroxaban through polymeric amorphous solid dispersion. Mol Pharmaceut. 2018;15(2):652-668. doi:10.1021/acs.molpharmaceut.7b01027

21. Wu L, Liu M, Shan W, et al. Bioinspired butyrate-functionalized nanovehicles for targeted oral delivery of biomacromolecular drugs. J Control Release. 2017;262:273-283. doi:10.1016/j. jconrel.2017.07.045

22. Zhou C, Xia X, Liu Y, Li L. The preparation of a complex of insulin-phospholipids and their interaction mechanism. J Pept Sci. 2012;18(9):541-548. doi:10.1002/psc.2423

23. Wu J, Zheng Y, Liu M, Shan W, Zhang Z, Huang Y. Biomimetic viruslike and charge reversible nanoparticles to sequentially overcome mucus and epithelial barriers for oral insulin delivery. $A C S$ Appl Mater Interfaces. 2018;10(12):9916-9928. doi:10.1021/ acsami.7b16524

24. Pangeni R, Choi JU, Panthi VK, Byun Y, Park JW. Enhanced oral absorption of pemetrexed by ion-pairing complex formation with deoxycholic acid derivative and multiple nanoemulsion formulations: preparation, characterization, and in vivo oral bioavailability and anticancer effect. Int $J$ Nanomedicine. 2018;13:3329-3351. doi:10.2147/IJN.S167958

25. Cheng MB, Wang JC, Li YH, et al. Characterization of water-inoil microemulsion for oral delivery of earthworm fibrinolytic enzyme. J Control Release. 2008;129(1):41-48. doi:10.1016/j. jconrel.2008.03.018

26. Halberg IB, Lyby K, Wassermann K, Heise T, Zijlstra E, PlumMorschel L. Efficacy and safety of oral basal insulin versus subcutaneous insulin glargine in type 2 diabetes: a randomised, double-blind, phase 2 trial. Lancet Diabetes Endocrinol. 2019;7(3):179-188. doi:10.1016/S2213-8587(18)30372-3 


\section{Supplementary materials}

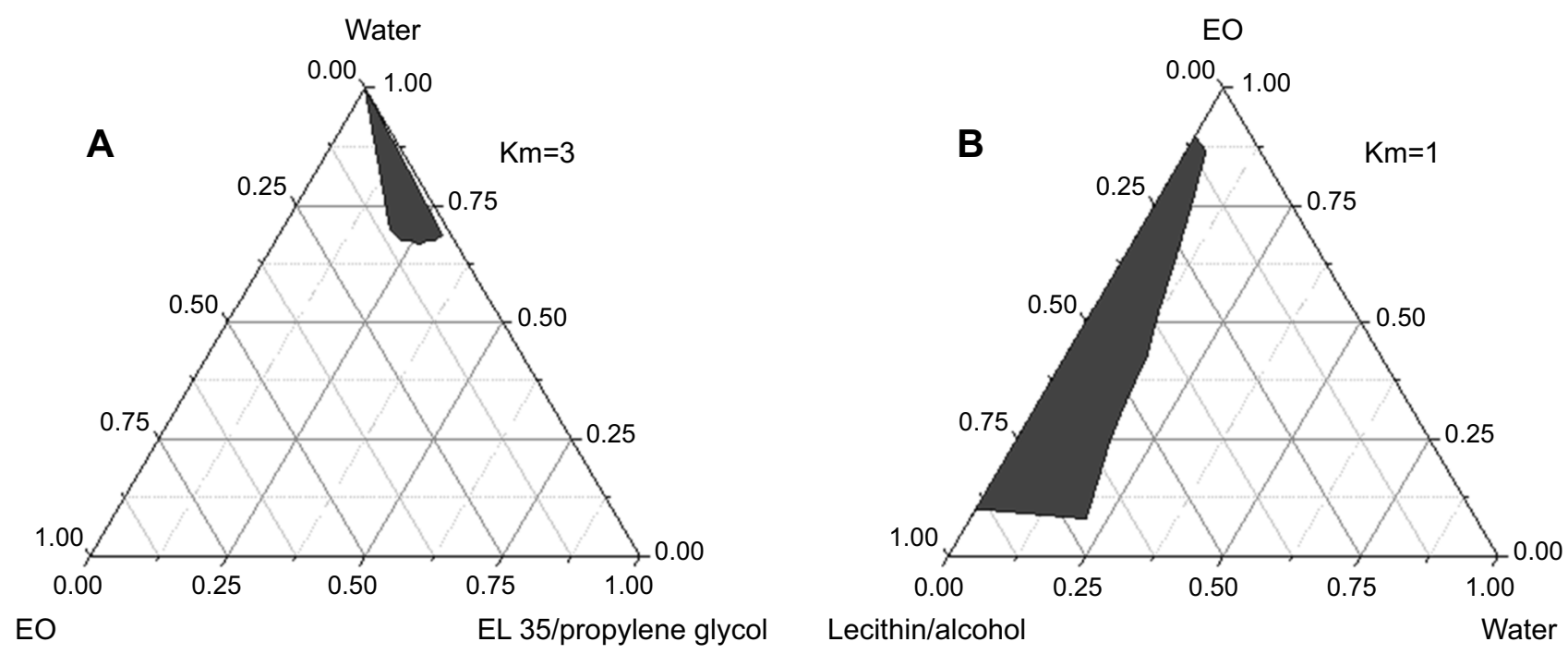

Figure SI Pseudo-ternary phase diagrams composed of (A) O/W nanoemulsion: Cremophor El and propylene glycol as surfactant and co-surfactant (3:I,w/w), (B) W/O nanoemulsion: lecithin and ethanol as surfactant and co-surfactant $(\mathrm{I}: \mathrm{I}, \mathrm{w} / \mathrm{W})$, EO was used as oil phase. The gray area represents the nanoemulsion range.

Abbreviations: EL35, Cremophor EL35; Km, the weight ratio of surfactant/co-surfactant; O/W, oil-in-water; W/O, water-in-oil..

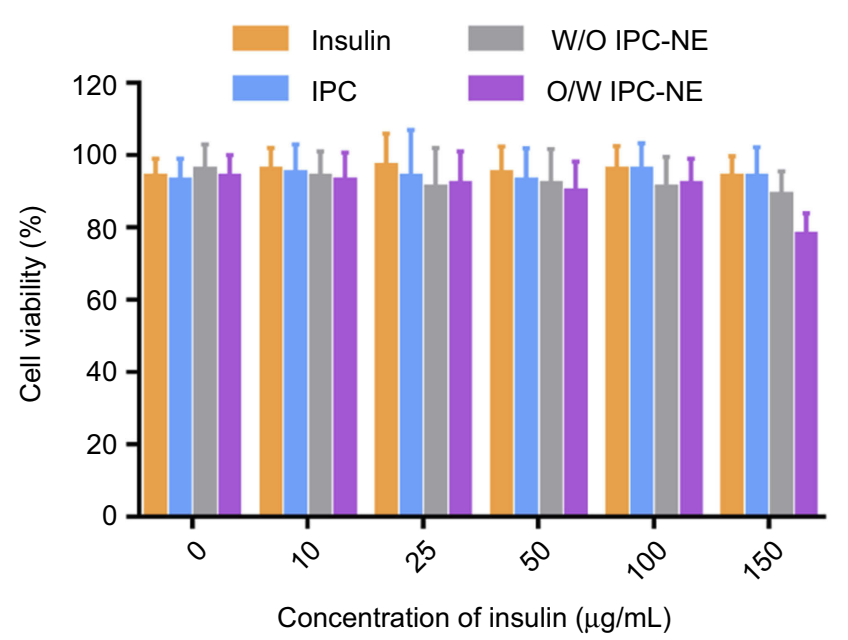

Figure S2 Cytotoxicity of insulin, IPC and IPC-NEs on Caco-2 cell treated at $37^{\circ} \mathrm{C}$ for 4 hrs. Data are present as mean \pm SD, $n=3$.

Abbreviations: IPC, insulin-phospholipid complex; IPC-NE, IPC-based nanoemulsion; O/W, oil-in-water; W/O, water-in-oil. 


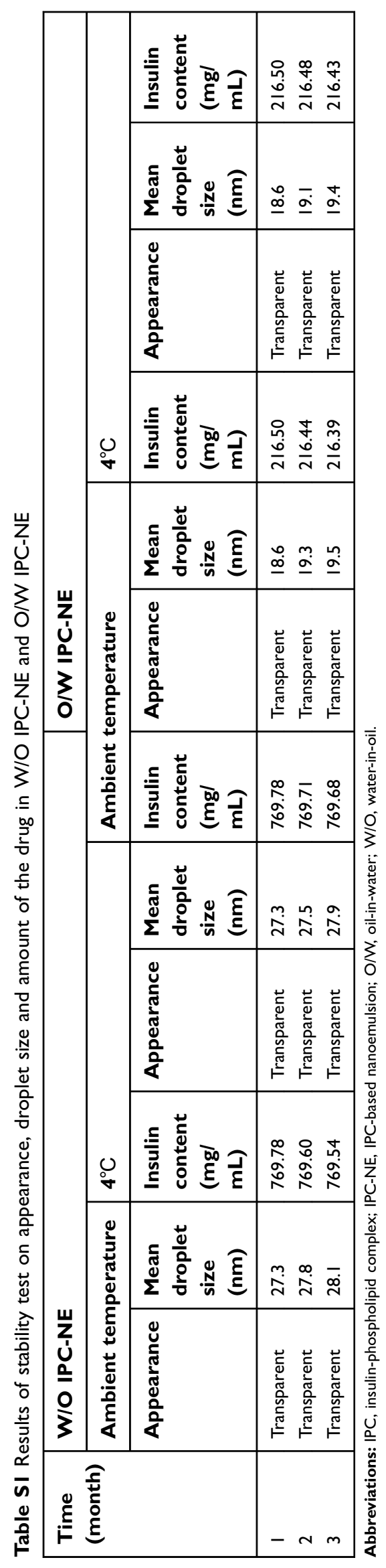




\section{Publish your work in this journal}

The International Journal of Nanomedicine is an international, peerreviewed journal focusing on the application of nanotechnology in diagnostics, therapeutics, and drug delivery systems throughout the biomedical field. This journal is indexed on PubMed Central, MedLine, CAS, SciSearch ${ }^{\circledR}$, Current Contents ${ }^{\circledR} /$ Clinical Medicine,
Journal Citation Reports/Science Edition, EMBase, Scopus and the Elsevier Bibliographic databases. The manuscript management system is completely online and includes a very quick and fair peer-review system, which is all easy to use. Visit http://www.dovepress.com/ testimonials.php to read real quotes from published authors.

Submit your manuscript here: https://www.dovepress.com/international-journal-of-nanomedicine-journal 